to appear in The Astrophysical Journal on March 20, 2002

\title{
Radio-to-FIR Spectral Energy Distribution and Photometric Redshifts for Dusty Starburst Galaxies
}

\author{
Min S. Yun \\ University of Massachusetts, Department of Astronomy, Amherst, MA 01003 \\ myun@astro.umass.edu \\ and \\ C. L. Carilli \\ National Radio Astronomy Observatory, P.O. Box 0, Socorro, NM 87801 \\ ccarilli@nrao.edu
}

\begin{abstract}
As a logical next step in improving the radio-to-submm spectral index as a redshift indicator (Carilli \& Yun), we have investigated a technique of using the entire radio-to-FIR spectral energy distribution (SED) for deriving photometric redshifts for dusty starburst galaxies at high redshift. A dusty starburst SED template is developed from theoretical understanding on various emission mechanisms related to massive star formation process, and the template parameters are selected by examining the observed properties of 23 IR selected starburst galaxies: $T_{d}=58 \mathrm{~K}, \beta=1.35$, and $f_{n t h}=1$. The major improvement in using this template SED for deriving photometric redshifts is the significant reduction in redshift uncertainty over the spectral index technique, particularly at higher redshifts. Intrinsic dispersion in the radio and FIR SEDs as well as absolute calibration and measurement errors contribute to the overall uncertainty of the technique. The derived photometric redshifts for five submm galaxies with known redshifts agree well with their spectroscopic redshifts within the estimated uncertainty. Photometric redshifts for seven submm galaxies without known spectroscopic redshifts (HDF850.1, CUDSS14.1, Lockman850.1, SMM J00266+1708, SMM J09429+4658, SMM J14009+0252, FIRBACK J1608+5418) are derived.
\end{abstract}

Subject headings: galaxies: high-redshift - galaxies: starburst - infrared: galaxies - submillimeter - radio continuum: galaxies - techniques: photometric 


\section{Introduction}

Sensitive observations at submm wavelengths are revealing what may be a population of active star forming galaxies at high redshift which are unseen in deep optical surveys due to dust obscuration (Smail et al. 1997; Barger et al. 1998; Hughes et al. 1998; Eales et al. 1999). The differential source counts clearly indicate a large excess of far-infrared sources by a factor of 10-50 over the no-evolution models derived from the optical deep survey data (Guiderdoni et al. 1998; Blain et al. 1999; Matsuhara et al. 2000; Scott et al. 2001), and a large fraction of star formation may be hidden by dust. The analysis of the submm SCUBA source counts and inferred redshift distribution based on the radio-submm flux density ratio (Carilli \& Yun 1999) suggests that luminous dusty galaxies may dominate the star formation history at early epochs $(z \sim 1-3)$ (Barger et al. 2000). Many of these faint submm sources are found to be very faint, red $(R \geq 25, K \geq 21)$ sources (Smail et al. 1999), whose optical redshifts may be inaccessible even for the 10-m class telescopes. These galaxies are nearly entirely missing from the optical studies of star formation at high redshifts (e.g. Steidel et al. 1999; Chapman et al. 2000), and a significant revision to the optically derived cosmic star formation history may be needed (see Blain et al. 1999).

Because direct redshift measurements are not possible in most cases, the redshift distribution and the cosmic evolution of the dusty submm galaxy population are not well determined at the moment. In a recent paper, we have proposed a technique of using the radio-to-submm spectral index as a redshift indicator. This technique is based on the universal radio-to-far infrared (FIR) correlation for star forming galaxies (Condon 1992), with the assumption that the spectral shapes may be similar enough to be able to differentiate between low and high redshift objects (see Carilli \& Yun 1999). To understand the magnitude of scatter in this relation and resulting uncertainty in the redshift estimates, we derived an empirical $\alpha_{1.4}^{350}-z$ relation $^{1}$ using the observed spectral energy distributions (SEDs) of 17 low redshift starburst galaxies (Carilli \& Yun 2000; also see Dunne, Clements, \& Eales 2000a). There is a significant scatter, but the existing data on high redshift star forming galaxies and AGN-hosts appear to follow this relation well.

While the radio-to-submm spectral index has been demonstrated to be a useful redshift indicator, using it to derive a redshift for any particular object may be risky because of the scatter in the observed $\alpha_{1.4}^{350}-z$ relation and the flattening in the relation at high redshifts. Obtaining redshift estimates using flux density ratios at other wavelength bands has also been suggested although none is particularly more successful (e.g. Hughes et al. 1998; Blain 1999; Hines \& Low 1999; Fox et al. 2001). More accurate redshift estimates for dusty

\footnotetext{
${ }^{1} \alpha_{1.4}^{350}$ is the spectral index between $1.4 \mathrm{GHz}$ and $350 \mathrm{GHz}$.
} 
starbursts may be obtained by utilizing the information associated with the entire radio-toFIR SED. Such a photometric redshift technique requires a good SED template, and here we derive an SED template based on the theoretical expectations of thermal dust, thermal Bremsstrahlung, and non-thermal synchrotron emission from a dusty starburst galaxy. Our SED template is constructed by examining the properties of the 23 IR selected starburst galaxies whose submm/FIR SED data are readily available in the literature. When applied to a sample of submm galaxies with known redshifts, the resulting redshift estimates are in excellent agreement with the spectroscopic redshifts. We also report photometric redshifts for a sample of submm galaxies without known spectroscopic redshifts, including the brightest SCUBA source in the Hubble Deep Field (Hughes et al. 1998).

\section{Starburst SED Model}

The observed SED from radio to infrared wavelengths for a dusty starburst galaxy is dominated by the energetic signatures of young stars and their environment. The bulk of the radiation from young OB stars is absorbed by dust and re-emitted in the infrared. The HII regions surrounding the young stars are also the sources of thermal Bremsstrahlung (freefree) emission. Energetic electrons produced in supernovae and their remnants are thought to account for nearly all of the radio synchrotron radiation. Following the discussion by Condon (1992), a simple starburst SED model is constructed as a linear sum of dust continuum $\left(S_{d}\right)$, thermal Bremsstrahlung or free-free $\left(S_{t h}\right)$, and non-thermal synchrotron $\left(S_{n t h}\right)$ emission.

\subsection{Thermal Dust Emission}

The flux density, $S_{d}(\nu)$, from a cloud at a distance $D_{L}$ containing $N$ spherical dust grains each of cross section $\sigma_{d}$, temperature $T_{d}$, and emissivity $Q(\nu)$, is given by

$$
S_{d}(\nu)=N\left(\sigma_{d} / D_{L}^{2}\right) Q(\nu) B\left(\nu, T_{d}\right),
$$

where $B\left(\nu, T_{d}\right)$ is the Planck function describing thermal black body radiation. The term $N\left(\sigma_{d} / D_{L}^{2}\right)$ is the solid angle $\Omega_{d}$ subtended by the dust source in the sky. For the emissivity function, we adopt a form

$$
Q(\nu)=1-\exp \left[-\left(\frac{\nu}{\nu_{c}}\right)^{\beta}\right]
$$

where $\beta$ is the dust emissivity index generally thought to be between 0 and 2 (Hildebrand 1983). This particular functional form allows the dust spectrum to be that of a purely black

body above the critical frequency $\nu_{c}$ where the dust clouds become optically thick while it is 
that of the $\nu^{2+\beta}$ grey body spectrum at lower frequencies. Observations suggest that thermal dust emission is nearly optically thick even at $\lambda \sim 100 \mu \mathrm{m}$ for galaxies dominated by intense

starburst regions (see Scoville et al. 1991; Solomon et al. 1997), and we adopt $\nu_{c}=2 \times 10^{12}$ $\mathrm{Hz}\left(\lambda_{c}=150 \mu \mathrm{m}\right)$. Combining the Eqs. $1 \& 2$,

$$
S_{d}(\nu)=\Omega_{d} B\left(\nu, T_{d}\right)\left(1-e^{-\left(\frac{\nu}{\nu_{c}}\right)^{\beta}}\right) .
$$

For a dust source of $\theta$ arcsec in diameter, expected flux density at $\nu$ (in $\mathrm{GHz}$ ) due to thermal dust emission is

$$
S_{d}(\nu)=2.8 \times 10^{-8} \frac{\nu^{3} \theta^{2}}{e^{\left[0.048 \nu / T_{d}\right]}-1}\left(1-e^{-\left(\frac{\nu}{2000}\right)^{\beta}}\right) \quad \mathrm{Jy} .
$$

The FIR luminosity, $L_{F I R}$, is derived by integrating Eq. 4 over the emitting area $\Omega_{d}$ and over the frequency range corresponding to $\lambda=40-500 \mu \mathrm{m}, L_{F I R}=4 \pi D_{L}^{2} \iint S_{d}(\nu) d \nu d \Omega$. The massive star formation rate (SFR) for a dusty starburst galaxy is then directly related to its dust continuum spectrum by the FIR luminosity (i.e. $S F R=L_{F I R} /(5.8 \times$ $\left.10^{9} L_{\odot}\right) M_{\odot} \mathrm{yr}^{-1}$, Kennicutt 1998)

\subsection{Thermal Bremsstrahlung (free-free) Emission}

Thermal Bremsstrahlung (free-free) emission from ionized gas in the HII regions surrounding hot young stars can be described in the same functional form as the thermal dust emission as in Eq. 3,

$$
S_{f f}(\nu)=\Omega_{f f} B\left(\nu, T_{e}\right)\left(1-e^{-\tau_{f f}}\right),
$$

where $T_{e}$ is electron temperature and $\tau_{f f}$ is free-free optical depth at the observed frequency. In the Rayleigh-Jeans regime $(h \nu \ll k T), B\left(\nu, T_{e}\right)=2 k T_{e} \nu^{2} / c^{2}$ at radio frequencies, and

$$
S_{f f}(\nu)=2 k T_{e} \nu^{2} c^{-2} \Omega_{f f}\left(1-e^{-\tau_{f f}}\right) .
$$

Free-free optical depth is given by $\tau_{f f}=0.083 E M \nu^{-2.1} T_{e}^{-1.35}$, where $E M \equiv \int n_{e}^{2} d l$ $\left(\mathrm{cm}^{-6} \mathrm{pc}\right)$ is the emission measure. In the optically thin regime, thermal Bremsstrahlung emission then has the familiar $\nu^{-0.1}$ dependence. A circular source of $\theta$ arcsec in diameter would have flux density of

$$
S_{f f}(\nu)=4.8 \times 10^{-8} E M \nu^{-0.1} T_{e}^{-0.35} \theta^{2} \quad \mathrm{Jy} .
$$




\subsection{Non-thermal Synchrotron Emission}

For an ensemble of relativistic electrons with a power-law distribution of energy $N(E)=$ $N_{\circ} E^{-\gamma}$ and an isotropic velocity distribution, their synchrotron emission coefficient is

$$
\epsilon \propto N_{\circ}(B \sin \theta)^{(\gamma+1) / 2} \nu^{(1-\gamma) / 2}
$$

where $\theta$ is the angle between the magnetic field $B$ and the line of sight to the observer. The resulting power-law spectrum has a form $S_{\nu} \propto \nu^{-\alpha}$ where $\alpha=(\gamma-1) / 2$ is the spectral index. Bell (1978) proposed the acceleration of cosmic rays by supersonic shocks (of velocity $v_{s}$ ) as the main mechanism for non-thermal synchrotron emission from supernova remnants and derived the volume emissivity as,

$$
\epsilon(\nu) \sim 3 \times 10^{-33} \nu^{-\alpha}\left(\frac{n}{c m^{-3}}\right)\left(\frac{\alpha}{0.75}\right)\left(\frac{v_{s}}{10^{4} k m / s}\right)^{4 \alpha}\left(\frac{B}{10^{-4} G}\right)^{\alpha+1} \operatorname{erg~s}^{-1} \mathrm{~Hz}^{-1} \mathrm{~cm}^{-3}
$$

Assuming supernova remnants dominate the non-thermal synchrotron emission from galaxies, Condon (1992) derived a relation $S_{n t h} \propto \nu_{S N}$ where $\nu_{S N}$ is the Type II supernova rate per year (see his Eq. 17). However, this relation predicts a $\nu_{S N}$ more than an order of magnitude too large for our Galaxy, and Condon proposed a revised relation based on Galactic normalization (i.e. his Eq. 18), which can be re-written as

$$
S_{n t h}(\nu)=1150 \nu^{-\alpha} \nu_{S N} D_{L}^{-2} \mathrm{Jy}
$$

where $D_{L}$ is luminosity distance in Mpc.

\subsection{Starburst SED Template}

The first and the foremost important step in all photometric redshift technique is establishing a robust and reliable template. Key SED template parameters for the starburst SED template are $T_{d}$ and $\beta$ for the dust emission (Eq. 4), $T_{e}$ and $E M$ for thermal free-free emission (Eq. 7), and $\nu_{S N}$ and $\alpha$ for the non-thermal synchrotron emission (Eq. 10). These parameters and their dispersions are determined by deriving best fit SED models for the 23 IR selected starburst galaxies whose submm/FIR data are readily available in the literature.

Flux density for dust emission $S_{d}$ can be derived directly using Eq. 3 by specifying the source size $\Omega_{d}$, dust temperature $T_{d}$, and emissivity $\beta$. The effective source solid angle $\Omega_{d}$ for a given source is derived from the observed $100 \mu \mathrm{m}$ flux density, assuming dust emission is nearly optically thick (see $\S 2.1$ ). For example, the prototypical ultraluminous galaxy Arp 220 is a $114 \mathrm{Jy}\left(1.14 \times 10^{-21} \mathrm{erg} \mathrm{cm}^{-2} \mathrm{~Hz}^{-1}\right)$ source at $100 \mu \mathrm{m}$, which translates to 
$\Omega_{d}=3 \times 10^{-11} \mathrm{rad}^{2}$ for $T_{d}=59 \mathrm{~K}$ (see Table 1 ). This corresponds to a circular area with $1.2^{\prime \prime}$ $(390 \mathrm{pc})$ in diameter ${ }^{2}$, which agrees well with the size of the molecular gas complex fueling the nuclear starburst traced in CO (Scoville, Yun, \& Bryant 1997; Downes \& Solomon 1998; Sakamoto et al. 1999). The best fit dust SED model is then selected by examining the parameter space for $T_{d}$ and $\beta$, between $25-85 \mathrm{~K}$ and 1.0-2.0, respectively.

Thermal Bremsstrahlung or free-free flux densities can be derived from Eq. 7 by determining the frequency where free-free opacity $\tau_{f f}$ becomes unity and estimating the size of the emitting region. Alternatively, one can also compute the free-free flux density from the inferred SFR as it is proportional to the production rate of Lyman continuum photons. Using the $\mathrm{H} \alpha$ normalization of Kennicutt (1998) for a Salpeter IMF and assuming $50 \%$ of Lyman continuum photons are quenched by dust absorption, Eq. 23 of Condon (1992) can be re-written as

$$
L_{f f}(\nu)=7.9 \times 10^{26} \nu^{-0.1}\left(\frac{S F R}{M_{\odot} y r^{-1}}\right) \operatorname{erg~s}^{-1} \mathrm{~Hz}^{-1} .
$$

The free-free flux density for a galaxy with a given FIR derived star formation rate is then

$$
S_{f f}(\nu)=0.71 \nu^{-0.1}\left(\frac{S F R}{M_{\odot} y r^{-1}}\right) D_{L}^{-2} \mathrm{Jy} .
$$

The free-free flux density $S_{f f}$ determined this way is nearly identical to the value derived using Eq. 7 assuming $\tau_{f f} \sim 1$ at $\nu=1 \mathrm{GHz}$ and $\Omega_{f f}=\Omega_{d}$.

Non-thermal synchrotron flux density $S_{n t h}$ can also be parameterized as a function of SFR since $\nu_{S N} \propto S F R$. Adjusting Eq. 20 of Condon (1992) for the Salpeter IMF, Eq. 10 can be re-written as,

$$
S_{n t h}(\nu)=25 f_{n t h} \nu^{-\alpha}\left(\frac{S F R}{M_{\odot} y r^{-1}}\right) D_{L}^{-2} \mathrm{Jy} .
$$

The synchrotron spectral index $\alpha$ is known to lie within a narrow range around $0.7-0.8$, and we simply adopt $\alpha=0.75$ to minimize the number of free parameters in our model. Condon's original derivation arbitrarily employed a Galactic normalization as noted earlier. We thus add a scaling factor $f_{n t h}$ (of order unity) here in order to determine the normalization more suitable for starburst galaxies.

Only the SED data at frequencies near and below the dust peak are used for the model fit because the mid- to near-IR data points clearly require additional, higher temperature dust components. The SED fit for Arp 220 is shown in Figure 1, and it demonstrates the effects of changing $T_{d}$ while holding $\beta$ constant. The scatter of data points about various

\footnotetext{
${ }^{2} \mathrm{An}$ ensemble of distributed sources with the equivalent total area is also allowed by this simple model.
} 
models suggests that there are systematic scaling differences among different flux density measurements, generally larger than the nominal uncertainties reported, especially in the submillimeter wavelengths. At a glance, all three dust temperature models plotted seem to do an adequate job of fitting the submm and FIR data points qualitatively. However, a closer examination reveals that the $T_{d}=74 \mathrm{~K}$ model fails to match the submm points. The $T_{d}=43 \mathrm{~K}$ model matches the submm data points reasonably well, but it clearly falls short on predicting the FIR data points. Previous studies of submm dust properties for luminous infrared galaxies generally favored cold dust temperature between 40 and $50 \mathrm{~K}$ for Arp 220 (e.g. Eales et al. 1989; Scoville et al. 1991; Dunne et al. 2000b). If we were to choose a single temperature dust SED model that best fits all observed data points between $1 \mathrm{~mm}$ and 60 $\mu \mathrm{m}$, however, a slightly warmer dust model is favored.

Our starburst SED model analysis is applied to 23 IR-selected starburst galaxies that are selected for (1) their $L_{F I R}$ exceeding $10^{11} L_{\odot}$; and (2) having at least two measurements covering their submm part of the spectrum. The SED data are primarily drawn from the IRAS Faint Source Catalog, NRAO/VLA Sky Survey (Condon et al. 1998), Condon et al. (1991), Carico et al. (1992), Rigopoulou et al. (1996), Benford (1999), Lisenfeld et al. (2000), Dunne et al. (2000b), and Dunne \& Eales (2001). As summarized in Table 1, the characteristic dust temperature $T_{d}$ ranges between $46 \mathrm{~K}$ and $74 \mathrm{~K}$, with a mean of $58 \pm 9 \mathrm{~K}$. Dust emissivity $\beta$ also ranges widely, between 1.05 and 1.70 , with a mean of $1.32 \pm 0.17$ and a median of 1.35 (see Figure 2). It is likely that all galaxies consist of an ensemble of dust clouds with a range of temperature (see Yun \& Scoville 1998; Frayer et al. 1999; Dunne \& Eales 2001), and single temperature SED models tend to favor a smaller $\beta$ (flatter submm dust spectrum) and higher dust temperature than typically found in giant molecular clouds (10-20 K). Since available SED measurements are generally sparse in practice, we accept these limitations of a single temperature dust model for the purpose of keeping the number of free parameters manageably small.

Thermal Bremsstrahlung (free-free) emission makes significant contribution only in the bottom of the SED trough between the non-thermal synchrotron and thermal dust feature, and it plays essentially no role in defining the starburst SED template. Variations in the nonthermal synchrotron emission are tracked by a parameter $f_{n t h}$ (see Eq. 13). The dispersion in the radio-FIR correlation is measured to be about 0.25 in a logarithmic scale (e.g. Yun et al. 2001), and a rather broad range of $f_{n t h}$ found (see Figure 3 ) is consistent with this expectation. Presence of a radio AGN (e.g. Mrk 231, NGC 6240) can account for the increase in some cases, but synchrotron emission efficiency may indeed vary from galaxy to galaxy. The median for the whole sample is 1.1, but it is reduced to 1.0 if the two clear radio AGN hosts are removed. Therefore, on average, the non-thermal synchrotron emission from these dusty starbursts appear to follow the Galactic normalization adopted by Condon (1992). 
In summary, we adopt $T_{d}=58 \mathrm{~K}, \beta=1.35$, and $f_{n t h}=1.0$ for our photometric redshift SED template. This relatively flat $\beta$ is in good agreement with the analysis of 102 galaxies by Dunne et al. (2000b), but the mean dust temperature we adopt is significantly warmer than the mean of $T_{d}=36 \pm 5 \mathrm{~K}$ Dunne et al. derived using $60 \mu, 100 \mu$, and $850 \mu$ measurements. The main difference is that the dust emission from our IR selected, luminous dusty starbursts are on average significantly warmer than those of the Dunne et al. sample, which includes a large number of late type field galaxies.

\section{Photometric Redshifts for Dusty Galaxies at High Redshift}

\subsection{Procedure}

Once the dusty starburst SED template is chosen, then the entire radio-to-FIR continuum spectrum for any given galaxy can be described in terms of just the total star formation rate $S F R$ and the luminosity distance $D_{L}$. The dust spectrum $S_{d}$ in Eq. 4 can be re-written as

$$
S_{d}(\nu)=1.5 \times 10^{-6} \frac{S F R(1+z) \nu^{3}}{D_{L}^{2}\left(e^{0.00083 \nu}-1\right)}\left(1-e^{-\left(\frac{\nu}{2000}\right)^{1.35}}\right) \quad \mathrm{Jy} .
$$

Combined with the expressions for free-free and non-thermal synchrotron emission given in Eqs. 12 \& 13, the entire radio-to-FIR continuum spectrum can be written as,

$$
S\left(\nu_{o b s}\right)=\left[25 f_{n t h} \nu_{\circ}^{-\alpha}+0.71 \nu_{\circ}^{-0.1}+1.5 \times 10^{-6} \frac{\nu_{\circ}^{3}\left(1-e^{\left.-\left(\frac{\nu_{\circ}}{2000}\right)^{1.35}\right)}\right.}{e^{0.00083 \nu_{\circ}}-1}\right] \frac{(1+z) S F R}{D_{L}^{2}} \mathrm{Jy},
$$

where $S F R$ is in $M_{\odot} \mathrm{yr}^{-1}, D_{L}$ in $\mathrm{Mpc}^{3}$, and $\nu_{\circ}=(1+z) \nu_{o b s}$ in $\mathrm{GHz} . \mathrm{A}(1+z)$ term is needed for sources at cosmological distances in order to account for the frequency or wavelength folding by the Doppler effect.

The redshift $z$ and the FIR luminosity $(S F R)$ of a particular galaxy can be determined simultaneously by comparing the observed SED with the dusty starburst template in the $(z, S F R)$ space. To be precise, one needs to include a correction term accounting for the cosmic microwave background (CMB) because most SED data are measured in contrast to the CMB. In practice this correction can be safely ignored as long as $T_{d} /(1+z) \gg 2.7 \mathrm{~K}$.

As a galaxy is placed further and further away, its entire SED shifts to the bottom (fainter, because of $D_{L}^{-2}$ ) and to the left (lower frequency, $\left.\nu_{o b s}=\nu_{o} /(1+z)\right)$ in the $(z, S F R)$

\footnotetext{
${ }^{3}$ We adopt $H_{\circ}=75 \mathrm{~km} \mathrm{sec}^{-1} \mathrm{Mpc}^{-1}$ and $q_{\circ}=0.5$ for this paper.
} 
space. This generic behavior for a dusty starburst and the resulting change in the apparent spectral index between $1.4 \mathrm{GHz}$ and $850 \mu \mathrm{m}$ has been pointed out previously as a redshift indicator by Carilli \& Yun (1999). The slope of the rising part of the dust spectrum is such that the Doppler shift of the spectrum nearly offsets the $D_{L}^{-2}$ drop in flux density, making the submm bands particularly attractive for blind searches, but the SED measurements on this part of the dust spectrum offer limited redshift information for the same reason (e.g. Hughes et al. 1998; Fox et al. 2001). As in other photometric redshift techniques, the redshift information comes from distinct spectral features such as the trough between the declining non-thermal synchrotron emission in radio and the sharp rise in the dust spectrum or the dust peak near the rest wavelength of $100 \mu \mathrm{m}$. Even when the dust peak in the FIR is not sampled by observations, the radio synchrotron measurements help set the vertical scale with respect to the dust spectrum, i.e. the $S F R$.

Because SED measurements for most submm galaxies include only a few discrete points rather than a continuous frequency coverage, our best fit SED model search utilizes a $\chi^{2}$ minimization with discrete sampling of the parameter space rather than a full cross-correlation technique. One advantage of this approach is that the upper limits in flux density can be incorporated in a straightforward way by simply rejecting all trial SEDs that are incompatible with the upper limits.

\subsection{Trials on Galaxies of Known Redshifts}

To test the robustness of our SED template, we apply this photometric redshift technique to several well studied submm galaxies, and these results are summarized in Table 2. Disregarding the formal uncertainties for the moment, the new photometric redshifts $z_{p h}$ are in excellent agreement with the spectroscopic redshifts $z_{s p}$ for the all five submm galaxies with known redshifts. When compared to the old radio-to-submm spectral index estimates $z_{S I}$ as shown in Figure 4, the improvement is seen mainly at high redshift $(z>2)$ where the effectiveness of the spectral index method diminishes due to the flattening of the $\alpha-z$ relation. Some improvement is generally expected for the new photometric method since more information is utilized. At the same time this comparison also highlights the efficiency of the spectral index technique which utilizes rather limited amount of information.

The best fit models are shown in Figure 5 for the four cases for which the most SED data exist. For the two well studied SCUBA sources SMM J02399-0136 ( $z=2.80$, Ivison et al. 1998) and SMM J14011+0252 ( $z=2.57$, Ivison et al. 2000), we derive photometric redshifts of $2.83 \& 2.73$, respectively. The agreement with their spectroscopic redshifts is good in both cases, and the improvement over the $\alpha-z$ estimate is quite substantial for 
SMM J02399-0136. The excellent agreement in SMM J02399-0136 is somewhat fortuitous since the $1.4 \mathrm{GHz}$ measurement is well above the best fit SED model (thus the source of a large reduced $\chi^{2}$ ), but the best fit solution is limited correctly by the $3 \sigma$ upper limit at 8.7 GHz. These two radio measurements are not consistent, and either the radio AGN is highly variable or the $1.4 \mathrm{GHz}$ measurement may be affected by source confusion. The photometric redshifts $\left(z_{p h}\right)$ for the submm galaxies SMM J02399-0134 ( $z=1.06$, Smail et al. 2000) and HR10 ( $z=1.44$, Cimatti et al. 1998; Dey et al. 1999) are also in good agreement with their spectroscopic redshifts within the uncertainty associated with the dust SED parameters (see $\S 4.4$ ). The SED fit for HR10 shown in Figure 5 suggests that the radio continuum is fainter than expected (i.e. $f_{n t h}<1$ ), which drove the $z_{p h}$ to a slightly higher value. Only in CUDSS14.18 ( $z=0.66$, Lilly et al. 1999) does the photometric redshift differ from the spectroscopic redshift by an amount larger than the nominal uncertainty of the technique. Only one submm SED measurement exists for this galaxy, and this may be an important limiting factor. CUDSS14.18 also appears to be slightly underluminous in radio continuum.

\subsection{Photometric Redshifts for Submm Galaxies}

Since the great majority of the submm galaxies are too faint to yield optical redshifts, a photometric technique may be the best way to infer their redshifts and evolution. We apply our photometric redshift technique to seven submm galaxies whose spectroscopic redshifts are unknown but are suspected of being at high redshifts: HDF850.1, Lockman850.1, CUDSS14.1, FIRBACK J1608+5418, SMM J00266+1708, SMM J09429+4658, SMM J14009+0252. The photometric redshifts and $S F R$ we derive are summarized in Table 2 , and the best fit models for six cases are shown in Figure 6 .

Perhaps the most interesting case is HDF850.1, which is the brightest SCUBA source in the Hubble Deep Field (Hughes et al. 1998; Downes et al. 1999a). Despite the extraordinarily deep HST imaging data available, this submm galaxy has eluded optical identification entirely. From the radio-to-submm flux density ratio, we have previously estimated its redshift to be $z>2.6$, but the flattening of the $\alpha-z$ relation at $z>2$ meant that its redshift was not very well constrained (Carilli \& Yun 1999). Our new photometric redshift analysis suggests that this galaxy has a high likelihood $\left(\chi_{n}^{2} \sim 1\right)$ of having a redshift near $z \sim 4.1$ with an intrinsic IR luminosity of about twice that of Arp 220. The uncertainty in the inferred redshift is too large $\left(\sigma_{z} \sim 0.5\right)$ for a $\mathrm{CO}$ search using existing instruments, but a spectroscopic verification of HDF850.1 and other optically faint submm galaxies should become possible using future broadband instruments on the Green Bank Telescope or the Large Millimeter Telescope. 
SMM J09429+4658 is another historically significant SCUBA galaxy, whose extremely dusty nature and red color $(K=19.4 \mathrm{mag}, I-K>6)$ was clearly demonstrated for the first time by the combined analysis of the high angular resolution VLA continuum imaging and deep near-IR imaging (Ivison et al. 2000). Our new photometric redshift analysis suggests a moderately high redshift $\left(z_{p h} \sim 3.9\right)$, but the SED model fit (Figure 6$)$ is quite poor $\left(\chi_{n}^{2} \sim 4\right)$ as our SED template cannot be fully reconciled with the three existing SED measurements. One possible explanation is that this submm galaxy is underluminous in radio continuum as in HR10 and CUDSS14.18, and its actual redshift is smaller.

SMM J00266+1708 is another clear example of an optically faint ( $K=22.5 \mathrm{mag})$ and red submm galaxy, whose extreme properties are clearly established by the combined analysis of the high angular resolution millimeter continuum observations at OVRO and deep nearIR imaging using the Keck telescope (Frayer et al. 2000). The derived photometric redshift of $z_{p h}=3.50$ makes it one of the highest redshift objects examined in this study, perhaps only the second after HDF850.1, and it has the second largest intrinsic FIR luminosity after SMM 02399-0136.

CUDSS14.1 is the brightest SCUBA source discovered in the CFRS 14hr field by Eales et al. (2000). Its optical counterpart has been identified using high angular resolution imaging at the VLA and IRAM interferometer (Eales et al. 2000; Gear et al. 2000), but it has not yet yielded a spectroscopic redshift. All four significant detection points lie along the model SED while all upper limits are consistent with the model. The derived photometric redshift of $z_{p h}=2.06$ is in good agreement with its optical photometric redshift of $z \sim 2$ (Lilly et al. 1999). Using the SED model of Dunne et al. (2000b) and optical to near-IR color, Gear et al. (2000) estimate its redshift to lie between 2 and 4.5 .

Lockman850.1 is the brightest SCUBA source detected in the Lockman Hole ISOPHOT survey region with an extremely red $(K \sim 20, I-K>6.2)$ and extended optical counterpart (Lutz et al. 2001). Comparing the observed SED to that of Arp 220, Lutz et al. estimate its redshift to be around 3, which agrees well with our photometric redshift $z_{p h}=2.72$.

SMM J14009+0252 is one of the submm sources identified in the Abell 1835 field by Ivison et al. (2000) with a faint optical counterpart $(K \sim 21)$. We derive $z_{p h}=1.30$, but the SED fit shown in Figure 6 for the dust spectrum is poor $\left(\chi_{n}^{2}=2.3\right)$. Ivison et al. rejected their own similarly modest redshift estimate because this galaxy is 3 times more luminous than HR 10 in the submm while it is more than 10 times fainter in $K$. Its $1.4 \mathrm{GHz}$ flux density is rather large for a SCUBA source, indicating a possible presence of a radio AGN. The SED fit for the dust spectrum alone suggests $z \sim 3.5$.

FIRBACK J1608+5418 is one of the FIR sources identified by the FIRBACK deep 
ISOPHOT survey (Dole et al. 2001). Incorporating its $350 \mu \mathrm{m}$ and $450 \mu \mathrm{m}$ detection at the CSO and the VLA detection at $1.4 \mathrm{GHz}$, Benford (1999) estimated its redshift to be around $z \sim 1.5$. Our photometric redshift of $z_{p h} \sim 0.8$ is substantially smaller. Its radiosubmm flux density ratio suggests a presence of a radio AGN, and it is likely that higher than expected radio continuum flux density has led to a poor fit $\left(\chi_{n}^{2}=17\right)$. Excluding the radio measurements, an SED analysis for the submm/FIR dust peak alone suggests $z \sim 0.7$ with about 4 times larger FIR luminosity than Arp 220.

\section{Discussions and Summary}

The above discussions of the SED modeling for the individual submm detected galaxies clearly indicate that systematic SED variation among the individual galaxies is the dominant factor over the statistical uncertainty associated with the SED measurements. Here we discuss in detail the nature and the magnitude of the systematic effects associated with the dust and radio emission as well as the SED sampling and the intrinsic calibration uncertainties in the measurements. Their combined contribution to the photometric redshift uncertainty is quantified in $\S 4.4$.

\subsection{Effects of Dust Temperature and Emissivity}

Doppler shift of a dust spectrum to a higher redshift is exactly equivalent to lowering of dust temperature, and departure in $T_{d}$ from the template SED translates directly to a redshift error (see Blain 1999, for further discussions). In other words, if the dust temperature of a galaxy is actually higher than our template, then the resulting $z_{p h}$ would be an under-estimate. While inherently subject to an object-to-object variation, the magnitude of uncertainty due to the spread in $T_{d}$ is well understood quantitatively: $\Delta z \sim \frac{\Delta T_{d}}{T_{d}}(1+z)$. If $T_{d}$ is higher or lower by $10 \mathrm{~K}$, the resulting error in the photometric redshift is about 0.3 at $z=1$, and it grows as $(1+z)$ reaching about 0.7 at $z=3$.

Dust emissivity $\beta$ is as important as dust temperature $T_{d}$ because it determines the location of the low frequency edge of the thermal dust feature in the SEDs. There is significant degeneracy between $\beta$ and $T_{d}$ (e.g. Dunne \& Eales 2001), and the decoupling the two becomes very difficult even when the dust spectrum is well sampled. In practice only a few measurements along the rising part of the dust SED are measured, and any deficiency in $T_{d}$ is at least partly offset by the compensating effect of $\beta$. A trend supporting this effect is seen in Figure 2 as a broad trend of decreasing $\beta$ with increasing $T_{d}$. A better demonstration of 
this effect is seen in Figure 1 where the submm SED points alone might be fit well by a dust spectrum with $T_{d} \sim 45 \mathrm{~K}$ and $\beta \sim 1.5$. The observed trend of increasing $T_{d}$ and deceasing

$\beta$ with increasing $S F R$ is also clearly seen in the theoretical modeling of dust heating and emission using a self-consistent 3-D radiative transfer code by Misselt et al. (2001). Therefore the error estimate based on the $T_{d}$ alone might serve only as a reasonable upper bound.

\subsection{Effects of the Radio Spectrum}

Another reason why the photometric redshift error based on the scatter in $T_{d}$ alone might be an over-estimate is that our SED fitting scheme relies as much on the spectral trough between the radio continuum and thermal dust emission as the dust spectrum itself. If this spectral trough is considered as the primary SED feature from which the photometric redshift information is derived, then it is easy to see that the variation in the dust spectrum is strongly modulated by the radio spectrum which is not affected.

The radio spectrum has its own uncertainty in its overall scaling as shown by the distribution of $f_{n t h}$ (see Figure 3), and its contribution to the photometric redshift error has already been alluded to in $\S 3.2$. Presence of a powerful radio AGN was a severe limiting factor for our earlier redshift estimation based on the radio-to-submm spectral index (Carilli \& Yun 1999), and it is not surprising that radio AGNs pose the biggest obstacle for the SED fitting photometric technique as well. The photometric redshifts derived for an ensemble of QSOs that are associated with dusty hosts are given at the bottom of Table 2 (also see Fig. 4). Although there are exceptions such as LBQS $1230+1627$ where $z_{p h} \sim z_{s p}$, the new photometric method does not fare much better than the spectral index method - the median $\chi_{n}^{2} \sim 7$.

In practice, radio AGNs are not likely to limit the photometric redshift technique utilizing a starburst SED template because only a small minority of the known submm galaxies show evidence of hosting a powerful AGN. Comparisons of deep X-ray and submm surveys have shown very little overlap between the detected sources (e.g. Fabian et al. 2000; Hornschemeier et al. 2000; Barger et al. 2001). At least three out of five submm galaxies discussed in $\S 3.2$ are known to be an AGN host, but the presence of an AGN also makes their spectroscopic redshift measurement possible and the use of a photometric technique unnecessary. The total fraction of infrared selected galaxies whose radio-to-FIR SED shows a clear sign of energetic AGN is only a few percent in the local universe (Yun et al. 2001), and this fraction appears to remain roughly the same at higher redshifts. 


\subsection{SED Sampling and Calibration}

Another important source of uncertainty is poor sampling of the SED. Sampling only the radio part of the SED offers virtually no redshift information, and the same is true if only the submm part of the SED is measured. On the other hand, because we are fitting the SED features changing over logarithmic scales, only a few well placed SED data points are needed to derived the redshifts (Carilli \& Yun 1999). More than one measurement on both side of the SED trough is highly desirable for a successful photometric redshift determination.

A related problem is the calibration and the relative weighting of individual SED measurements. Measurement uncertainties found in the literature often do not fully account for the overall calibration accuracy as demonstrated by the magnitude of the scatter and the sizes of the error bars plotted for the SED measurements of Arp 220 in Figure 1. For example, the IRAS $60 \mu \mathrm{m}$ and $100 \mu \mathrm{m}$ measurements found in the Point Source Catalog (PSC) and the Faint Source Catalog (FSC) are known to have systemic calibration differences of order $10 \%$ while the flux densities reported in the FSC often include uncertainties of only a few percent. For deriving the SED template from the 23 infrared selected starbursts, we adopted flux densities in the FSC but increased measurement uncertainties to 10\% (unless the reported uncertainty was larger). This re-calibration of the measurement uncertainty is more than cosmetic since the relative weight of the data points are directly reflected in the $\chi^{2}$ minimization. We found this re-weighting to be critically important in utilizing the submm

measurements. Similarly, all radio continuum measurements are assigned at least $10 \%$ uncertainty in order to account for the overall uncertainty in the flux calibration. No effort was made to re-calibrate any of the submm data points since most measurements generally carry relatively large fractional uncertainties, but some are clearly under-estimates based on the SED plots such as shown in Figure 1. The same problems also plague the reported SED measurements of many submm galaxies, and they will inevitably impact the accuracy of the photometric redshifts derived from these data. One way to improve the situation in the future would be employing a frequency selective bolometer (e.g. Kowitt et al. 1996; Meyer et al. 2001) that can make simultaneous measurements of several submm bands with accurate relative calibration between the measurement bands.

\subsection{Estimate of Uncertainty in the Radio-to-FIR SED Technique}

The uncertainty of $\sigma_{z} \sim 0.3(1+z)$ derived from the scatter in dust temperature is probably the upper bound to the error one may expect from the photometric technique using the dusty starburst SED template. The compensating effects of the dust emissivity and the radio continuum are more difficult to quantify as are the uncertainties contributed 
by the scatter in $f_{n t h}$. One way to estimate the collective uncertainty of the SED photometric technique is to apply this technique to the same 23 dusty starbursts from which the SED template was derived. This is not entirely circuitous since our SED template is based only on the average properties of these galaxies and has no knowledge of dispersions. At the least, we may be able to confirm the impact of the $10 \mathrm{~K}$ scatter in the dust temperature on the dispersion in $z_{p h}$ if dust temperature variation dominates the uncertainty in determining photometric redshifts.

The resulting "photometric redshift" $z_{p h}$ for the 23 dusty starbursts are listed in the last column of Table 1 . There are several galaxies with negative $z_{p h}$ since the impact of higher dust temperature for a $z=0$ galaxy with $T_{d}>58 \mathrm{~K}$ would result in $z_{p h}<0$. The $\chi^{2}$ minimization program is modified to search a redshift range of $-1 \geq z_{p h} \geq+1$ with modified scaling along the flux density axis in order to remove the non-physical impact of negative redshift on $D_{L}$. A handful of galaxies with negative redshift are known (e.g. M81), but negative redshift is generally considered non-physical. Here, the only physically meaningful interpretation of a negative $z_{p h}$ we derive is the measure of the magnitude of departure in the radio and dust property from the template SED, just the same way any uncertainty in $z_{p h}$ should be interpreted at any redshift.

A histogram of the resulting photometric redshifts shown in Figure 7 suggests that the median "redshift" is about +0.05 , suggesting the template SED may be slightly biased, but this offset is probably not very significant given the range of "redshifts" derived. "Photometric redshifts" as large as +0.3 and as small as -0.3 are found as expected from the scatter in $T_{d}$. The two extreme negative " $z_{p h}$ " object IRAS $08572+3915$ and Mrk 231 have $T_{d}$ of 74 $\mathrm{K}$ and $72 \mathrm{~K}$, respectively, following the general trend expected of the dust temperature variation. On the other hand, two other dusty starbursts with characteristic $T_{d} \geq 70 \mathrm{~K}$, IRAS $15250+3609$ and IRAS $05189-2524$, have " $z_{p h}$ " of +0.01 and +0.05 , clearly demonstrating that there are other compensating effects and that the scatter in $T_{d}$ alone does not dictate the overall photometric redshift uncertainty.

Regardless of the underlying causes, the "photometric redshifts" for $2 / 3$ of all galaxies lie within $\Delta z \leq 0.10$, and we estimate the collective uncertainty of the photometric redshift technique, including the variations in the radio and dust properties as well as the uncertainties in the $\chi^{2}$ minimization process, is about 0.1 as long as this technique is applied to luminous dusty starburst galaxies only. Allowing for the $(1+z)$ frequency folding of the Doppler effect, we estimate an overall uncertainty of $\sigma_{z} \sim 0.1(1+z)$ with an upper bound in redshift uncertainty of about $0.3(1+z)$.

In either case, this photometric redshift technique utilizing the radio-to-FIR dusty starburst SED represents a significant step forward, particularly at high redshifts, when com- 
pared with existing methods. The full potential of this method will be realized when the sources identified by several large deep, multi-frequency surveys planned in the immediate future (e.g. SIRTF Legacy Surveys) are analyzed together to reveal an accurate redshift distribution of luminous dusty galaxies at high redshift.

The National Radio Astronomy Observatory is a facility of the National Science Foundation operated under cooperative agreement by Associated Universities, Inc. Some of the data presented here are obtained from the NASA/IPAC Extragalactic Database (NED), which is operated by the Jet Propulsion Laboratory, California Institute of Technology, under contract with the National Aeronautical and Space Administration. 


\section{REFERENCES}

Andreani, P., Cimatti, A., Loinard, L., Rottgering, H. 2000, A\&A, 354, L1

Barger, A. J., Cowie, L. L., Sanders, D. B., Fulton, E., Taniguchi, Y. et al. 1998, Nature, 394,248

Barger, A., Cowie, L.L., \& Richards, E.A. 2000, AJ, 119, 2092

Barger, A. J., Cowie, L. L., Steffen, A. T., Hornschemeier, A. E., Brandt, W. N., Garmire, G. P. 2001, ApJ, 560, L23

Barvainis, R., Antonucci, R., Coleman, P. 1992, ApJ, 399, L19

Bell, A. R. 1978, MNRAS, 182, 443

Benford, D. J. 1999, PhD thesis, California Institute of Technology

Benford, D. J., Cox, P., Omont, A., Phillips, T. G. McMahon, R. G. 1999, ApJ, 518, L65

Blain, A. 1999, MNRAS, 309, 955

Blain, A., Smail, I., Ivison, R. J., \& Kneib, J.-P. 1999, MNRAS, 302, 632

Carico, D. P., Keene, J., Soifer, B. T., Neugebauer, G. 1992, PASP, 104, 1086

Carilli, C. L., \& Yun, M. S. 1999, ApJ, 513, L13

Carilli, C. L., \& Yun, M. S. 2000, ApJ, 530, 618

Chapman, S. C., Scott, D., Steidel, C. C., Borys, C., Halpern, M. et al. 2000, MNRAS, 319, 318

Cimatti, A., Andreani, P., Rottgering, H., Tilanus, R. 1998, Nature, 392, 895

Condon, J. J. 1992, ARAA, 30, 575

Condon, J. J., Huang, Z. P., Yin, Q. F., \& Thuan, T. X. 1991, ApJ, 378, L65

Condon, J. J., Cotton, W. D., Greison, E. W., Yin, Q. F., Perley, R. A. et al. 1998, AJ, 115, 1693

Dey, A., Graham, J. R., Ivison, R. J., Smail, I., Wright, G. S., Liu, M. C. 1999, ApJ, 519, 610

Dole, H., Gispert, R., Lagache, G., Puget, J.-L., Bouchet, F. R. et al. 2001, A\&A, 372, 364 
Downes, D., \& Solomon, P. M. 1998, ApJ, 507, 615

Downes, D., Neri, R., Greve, A., Guilloteau, S., Casoli, F. et al. 1999a, A\&A, 347, 809

Downes, D., Neri, R., Wiklind, T., Wilner, D. J., Shaver, P. A. 1999b, ApJ, 513, L1

Dunne, L., Clements, D. L., Eales, S. A. 2000, MNRAS, 319, 813

Dunne, L., Eales, S. A., Edmunds, M. G., Ivision, R. J., Alexander, P., Clements, D. L. 2000, MNRAS, 315, 115

Dunne, L., \& Eales, S. A. 2001, MNRAS, 327, 697

Eales, S. A., Wynn-Williams, C. G., \& Duncan, W. D. 1989, ApJ, 339, 859

Eales, S., Lilly, S., Gear, W., Dunne, L., Bond, J. R. et al. 1999, ApJ, 515, 615

Eales, S., Lilly, S., Webb, T., Dunne, L., Gear, W., Clements, D., Yun, M. 2000, AJ, 120, 2244

Fabian, A. C., Smail, I., Iwasawa K. et al. 2000, MNRAS, 315, L8

Fox, M. J., Efstathiou, A., Rowan-Robinson, M., Dunlop, J. S., Scott, S. et al. 2001, MNRAS, in press

Frayer, D. T., Ivison, R. J., Smail, I., Yun, M. S. \& Armus, L. 1999, AJ, 118, 139

Frayer, D. T., Smail, I., Ivison, R. J., \& Scoville, N. Z. 2000, AJ, 120, 1668

Gear, W. K., Lilly, S. J., Stevens, J. A., Clements, D. L., Webb, T. M., Eales, S. A., Dunne, L. 2000, MNRAS, 316, L51

Guiderdoni, B., Hivon, E., Bouchet, F.R., \& Maffei, B., 1998, MNRAS, 295, 877

Guilloteau, S., Omont, A., Cox, P., McMahon, R. G., Petitjen, P. 1999, A\&A, 349, 363

Hildebrand, R. H. 1983, QJRAS, 34, 267

Hines, D. C., Low, F. J. 1999, in Photometric Redshifts and the Detection of High Redshift Galaxies, eds. R. Weymann, L. Storrie-Lombardi, M. Sawicki, R. Brunner, ASP Conference Series, Vol. 191, p.265

Hornschemeier, A. E., Brandt, W. N., Garmire, G. P. et al. 2000, ApJ, 541, 49

Hughes, D. H., Serjeant, S., Dunlop, J., Rowan-Robinson, M., Blain, A. et al. 1998, Nature, 394,241 
Irwin, M. J., Ibata, R. A., Lewis, G. F., Totten, E. J. 1998, ApJ, 505, 529

Ivison, R. J., Smail, I., Le Borgne, J.-F., Blain, A. W., Kneib, J.-P. et al. 1998, MNRAS, 298,583

Ivison, R. J., Smail, I., Barger, A. J., Kneib, J.-P., Blain, A. W. et al. 2000, MNRAS, 315, 209

Kennicutt, R. C. 1998, ApJ, 498, 541

Knudsen, K. K., van der Werf, P. P., Jaffe, W. 2000, in Deep Millimeter Surveys: Implications for Galaxy Formation and Evolution, eds. J. Lowenthal and D. Hughes, World Scientific Publishers (astro-ph/0009024)

Kowitt, M. S., Fixsen, D. J., Goldin, A., Meyer, S. S. 1996, Appl. Opt., 35, 5630

Lewis, G. F., Chapman, S. C., Ibata, R. A., Irwin, M. J., Totten, E. J. 1998, ApJ, 505, L1

Lilly, S. J., Eales, S., Gear, W., Hammer, F., Le Favre, O. et al. 1999, ApJ, 518, 641

Lisenfeld, U., Isaak, K. G., Hills, R. 2000, MNRAS, 312, 433

Lutz, D., Dunlop, J. S., Almaini, O., Andreani, P., Blain, A. et al. 2001, A\&A, 378, 70

Matsuhara, H., Kawara, K., Sato, Y., Taniguchi, Y., Okuda, H. et al. 2000, A\&A, 361, 407

McMahon, R. G., Priddey, R. S., Omont, A., Snellen, I., Withington, S. 1999, MNRAS, 309, L1

Meyer, S. S., Cottingham, D., Crawford, T. C., Fixsen, D. J., Goldin, A., Kowitt, M. S., Van Howe, J., Wilson, G. W. 2001, in preparation

Misselt, K. A., Gordon, K. D., Clayton, G. C., Wolff, M. J. 2001, ApJ, 551, 277

Omont, A., McMahon, R. G., Cox, P., Kreysa, E., Bergeron, J. et al. 1996, A\&A, 315, 10

Rowan-Robinson, M., Efstathiou, A., Lawrence, A., Oliver, A., Taylor, A. et al. 1993, MNRAS, 261, 513

Rigopoulou, D., Lawrence, A., \& Rowan-Robinson, M. 1996, MNRAS, 278, 1049

Sakamoto, K., Scoville, N. Z., Yun, M. S., Crosas, M., Genzel, R., Tacconi, L. J. 1999, ApJ, 514,68

Scott, D., Lagache, G., Borys, C., Chapman, S. C., Halpern, M. et al. 2000, A\&A, 357, L5 
Scott, S. E., Fox, M. J., Dunlop, J. S., Serjeant, S., Peacock, J. A. et al. 2001, MNRAS, in press

Scoville, N. Z., Sargent, A. I., Sanders, D. B., \& Soifer, B. T. 1991, ApJ, 366, L5

Scoville, N. Z., Yun, M. S., \& Bryant, P. M. 1997, ApJ, 484, 702

Smail, I., Ivison, R, \& Blain, A. 1997, ApJ, 490, L5

Smail, I., Ivison, R. J., Kneib, J.-P., Cowie, L. L., Blain, A. W. et al. 1999, MNRAS, 308, 1061

Smail, I., Ivison, R. J., Owen, F. N., Blain, A. W., \& Kneib, J.-P. 2000, ApJ, 528, 612

Solomon, P. M., Downes, D., Radford, S. J. E., \& Barrett, J. W. 1997, ApJ, 478, 144

Steidel, C. C., Adelberger, K. L., Giavalisco, M., Dickinson, M., \& Pettini, M. 1999, ApJ, 519,1

Yun, M. S., \& Scoville, N. Z. 1998, ApJ, 507, 774

Yun, M. S., Carilli, C. L., Kawabe, R., Tutui, Y., Kohno, K. et al. 2000, ApJ, 528, 171

Yun, M.S., Reddy, N., \& Condon, J. 2001, ApJ, 554, 803 


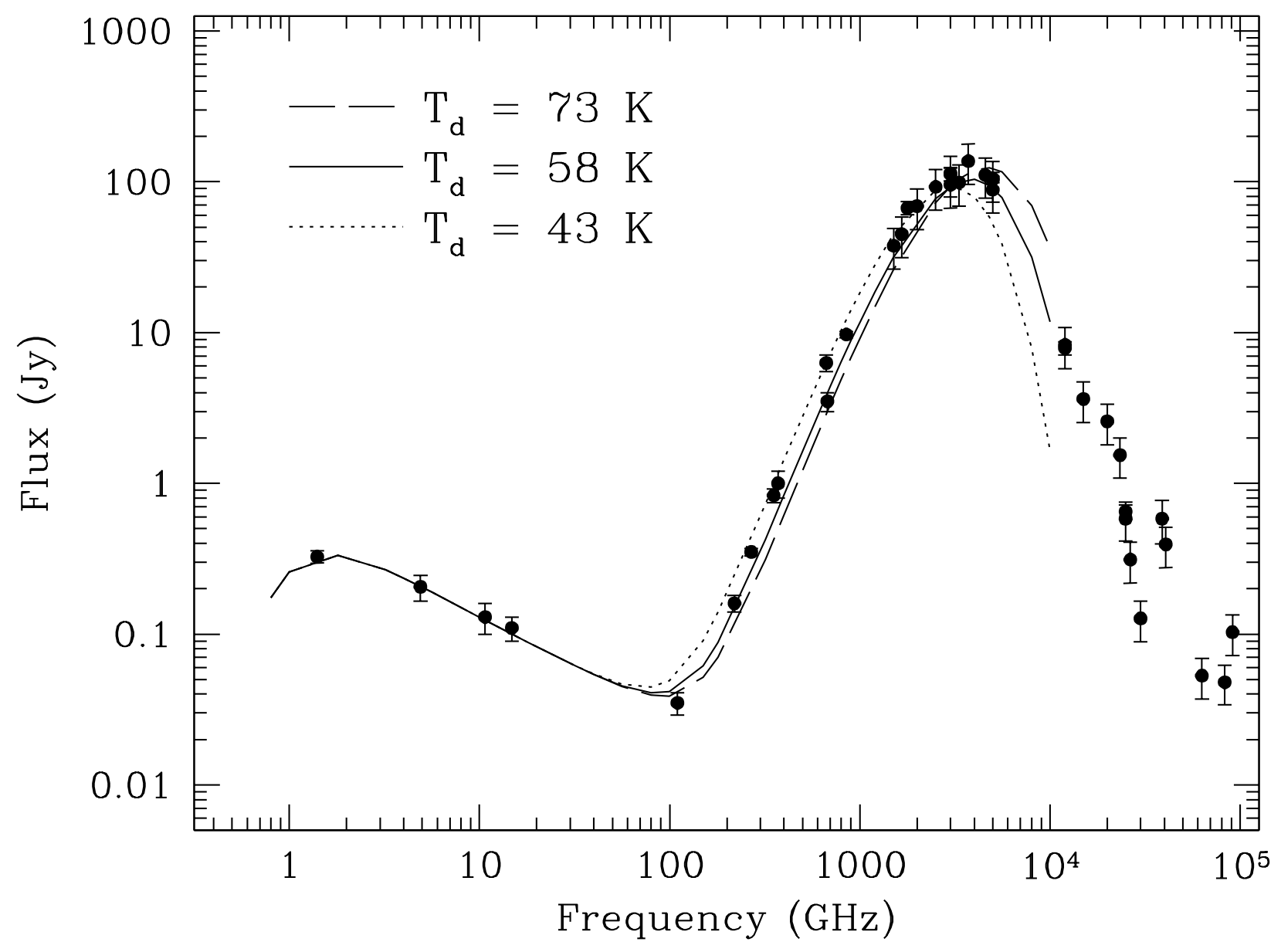

Fig. 1.- Radio-to-IR SED for Arp 220 is shown along with the model starburst SEDs with $\beta=1.35$ and $T_{d}=43 \mathrm{~K}, 58 \mathrm{~K}$, and $73 \mathrm{~K}$ are shown. The radio-FIR normalization term $f_{n t h}$ is set to 1.0, which is the Galactic normalization by Condon (1992). 


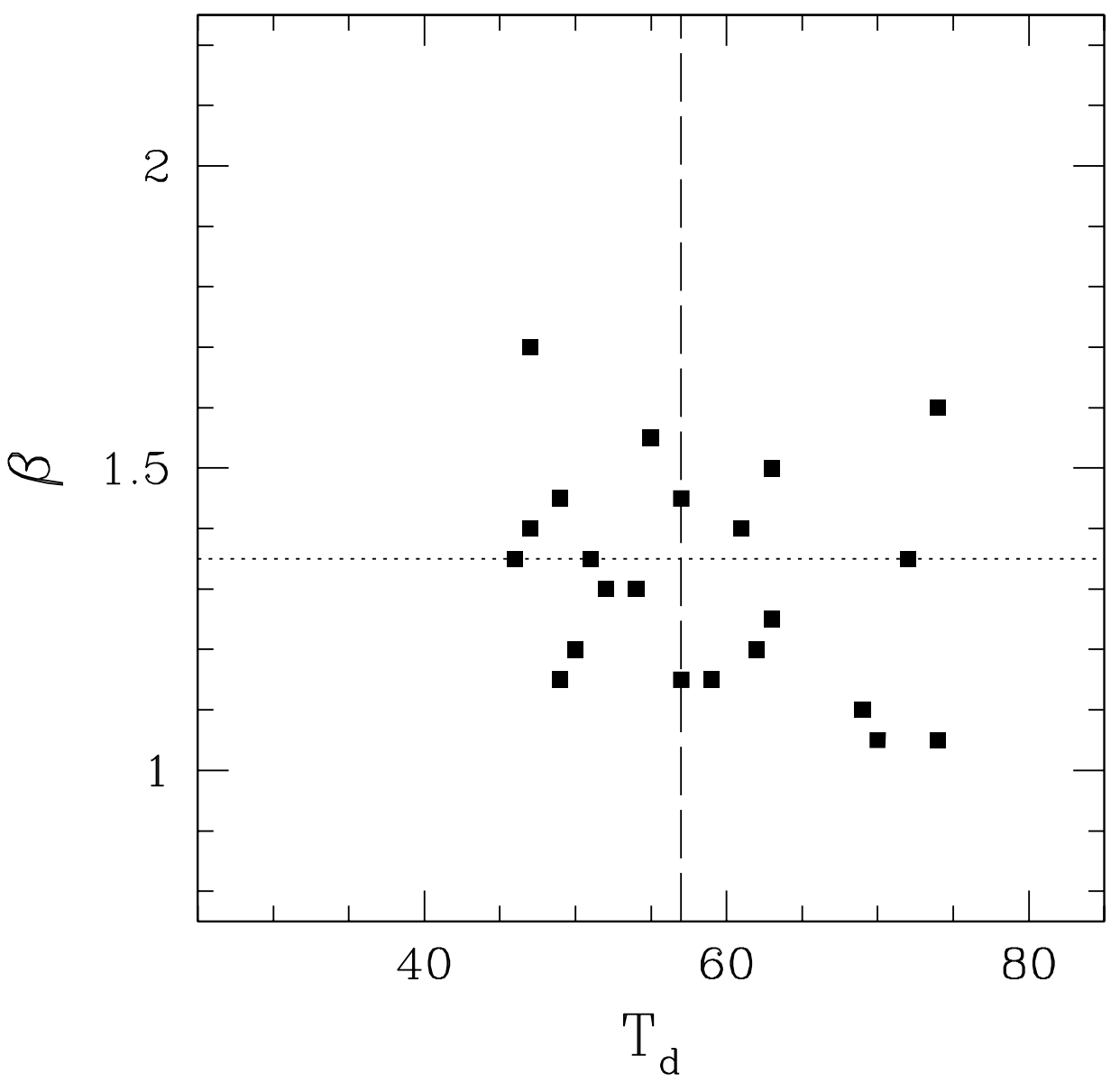

Fig. 2.- A distribution of best fit $\beta$ values versus $T_{d}$ for the 23 luminous IR starburst galaxies. The median values for $\beta$ and $T_{d}$ are $1.35 \& 57 \mathrm{~K}$ while the mean values are $1.32 \pm 0.17$ and $58 \pm 9 \mathrm{~K}$, respectively. 


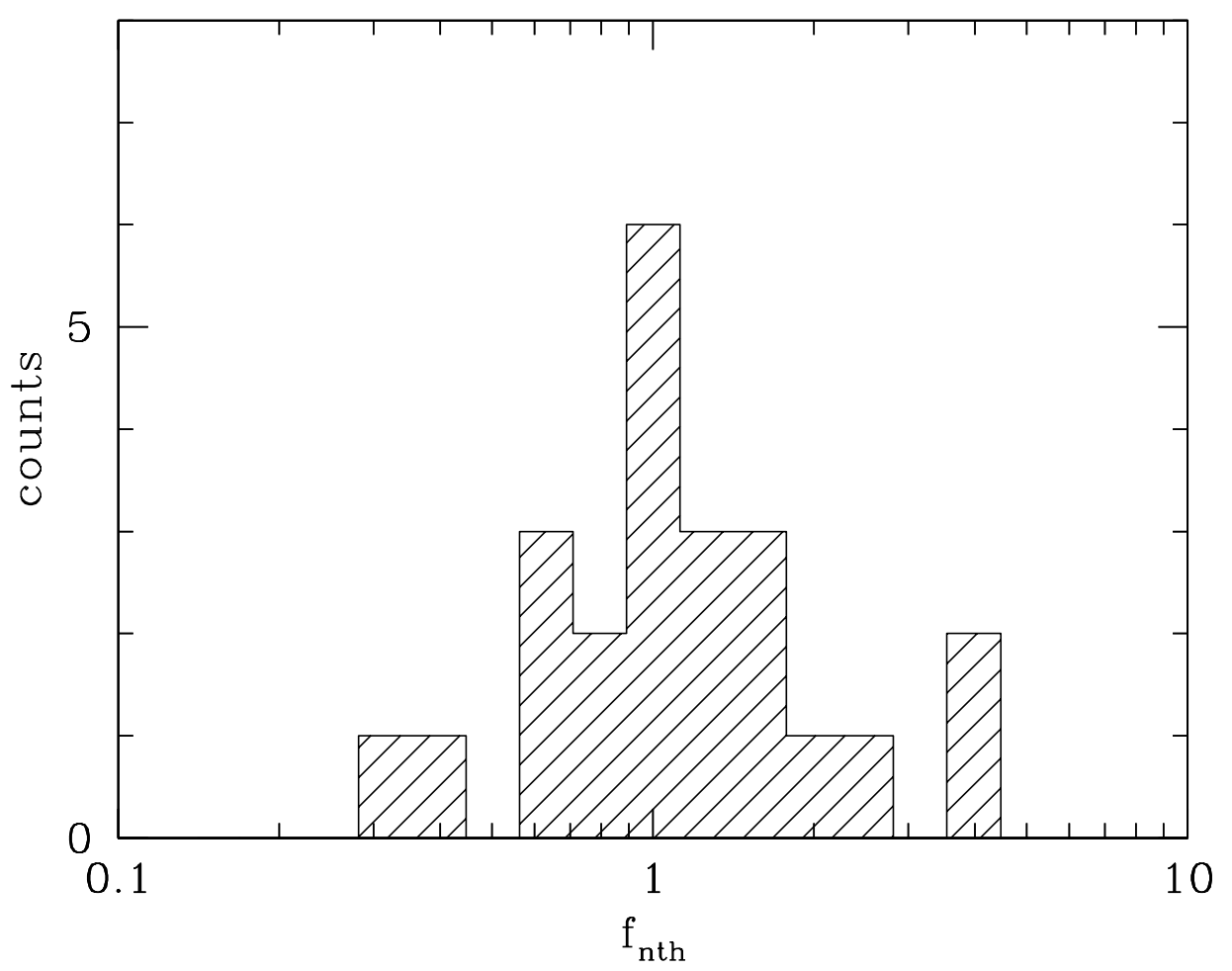

Fig. 3.- A distribution of $f_{n t h}$ values for the 23 luminous IR starburst galaxies. The median for the whole sample is 1.1, and it is reduced to 1.0 if two clear radio AGN hosts are removed. 

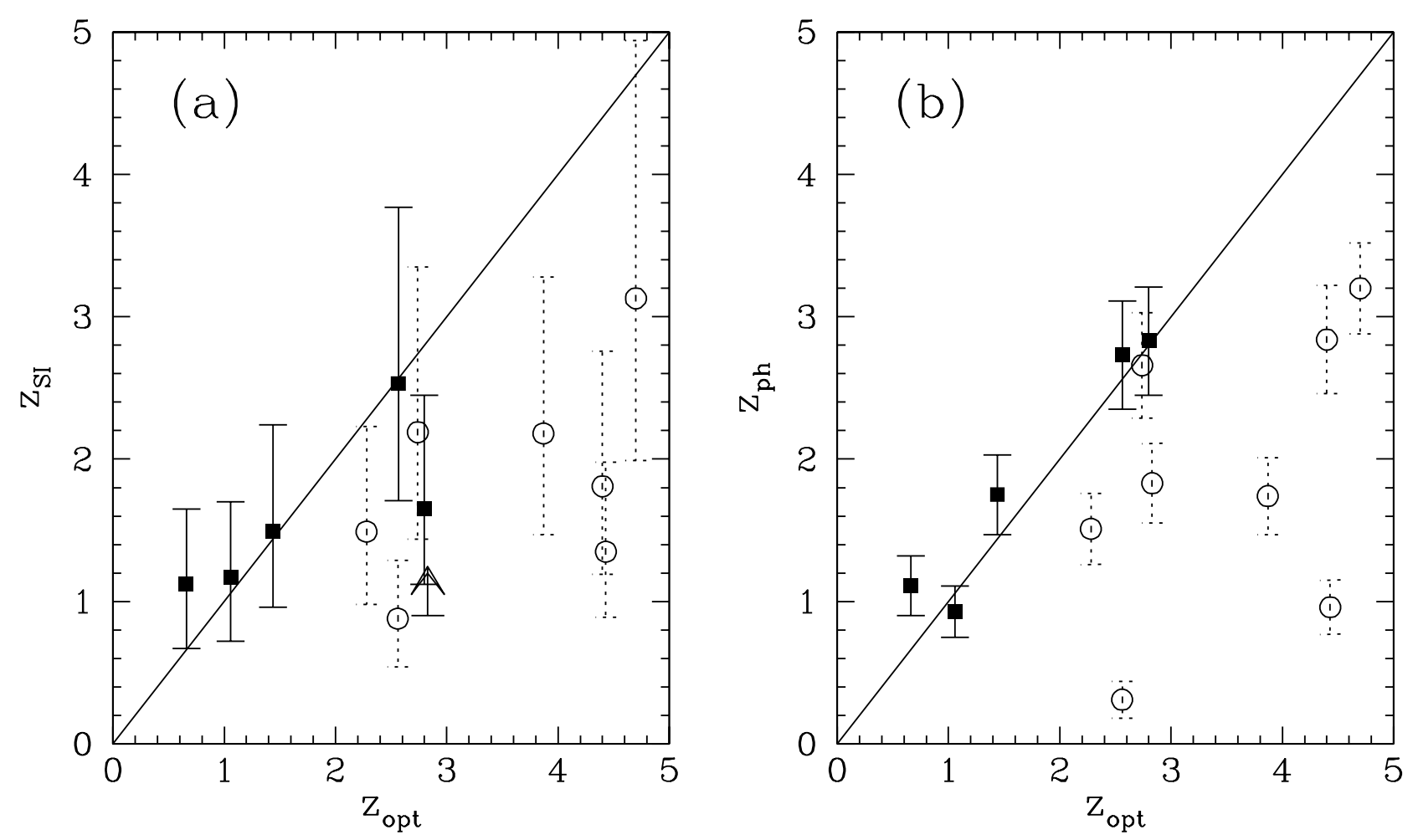

Fig. 4.- (a) A comparison plot of spectroscopic redshifts $\left(z_{\text {opt }}\right)$ versus redshift estimates from the radio-to-submm spectral index $\left(z_{S I}\right)$. (b) A comparison plot of spectroscopic redshifts $\left(z_{\text {opt }}\right)$ versus radio-to-FIR SED photometric redshifts $\left(z_{p h}\right)$. Solid squares represent submm galaxies while the empty circles are submm detected optical QSOs. 

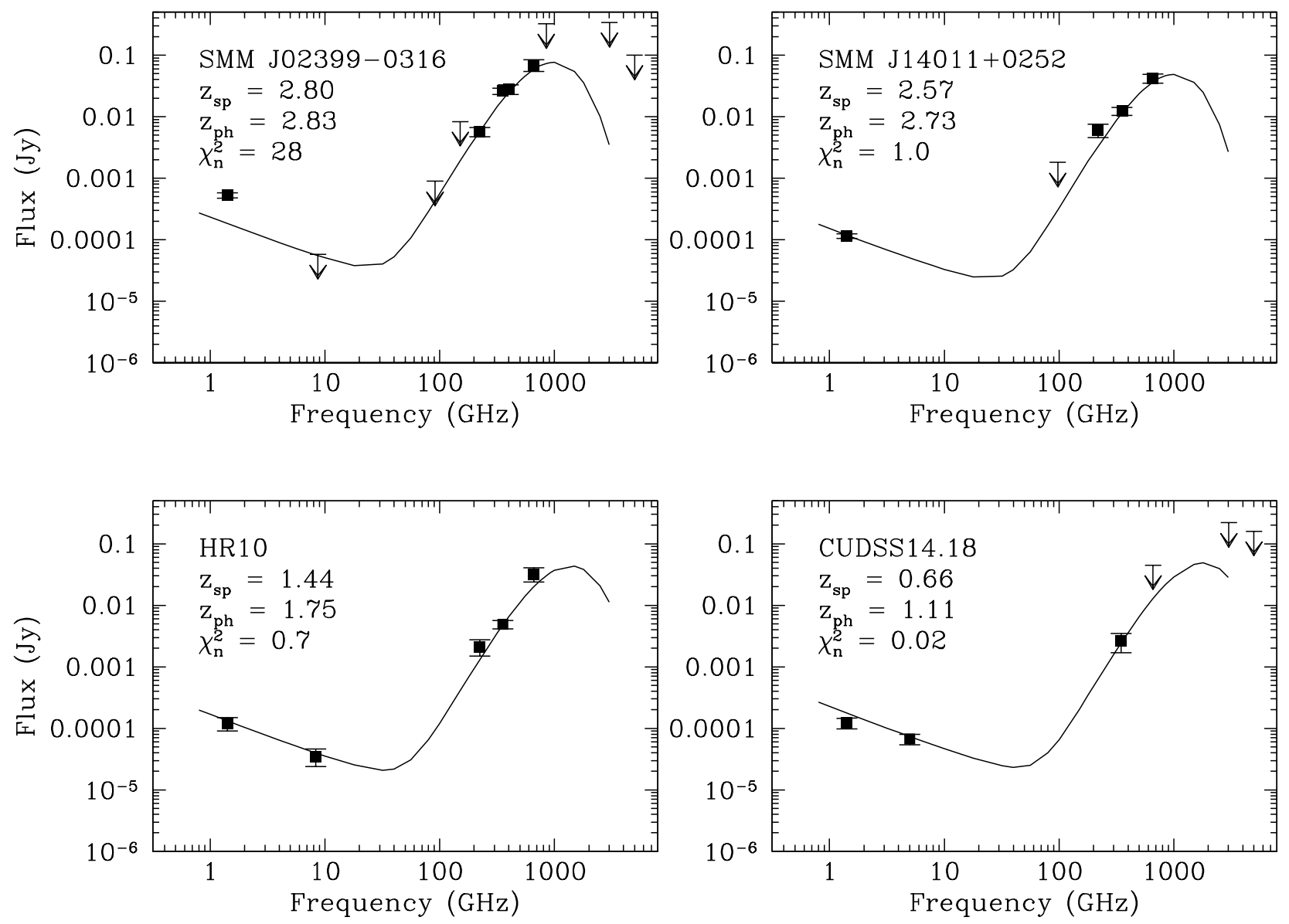

Fig. 5.- Results of the SED fits for four submm galaxies with known spectroscopic redshifts. 

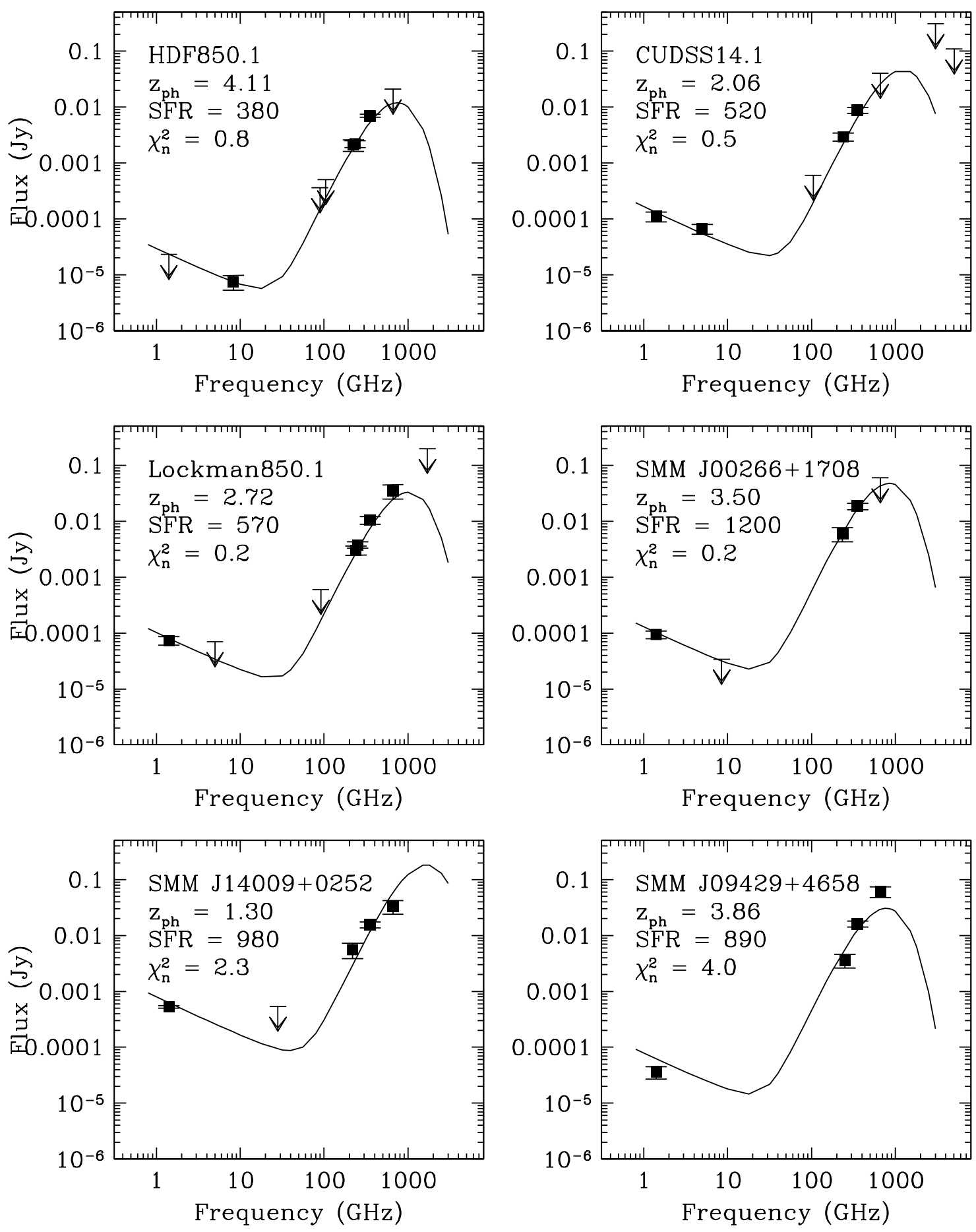

Fig. 6.- Results of the SED fits for six submm galaxies with unknown redshifts are shown, including HDF850.1. 


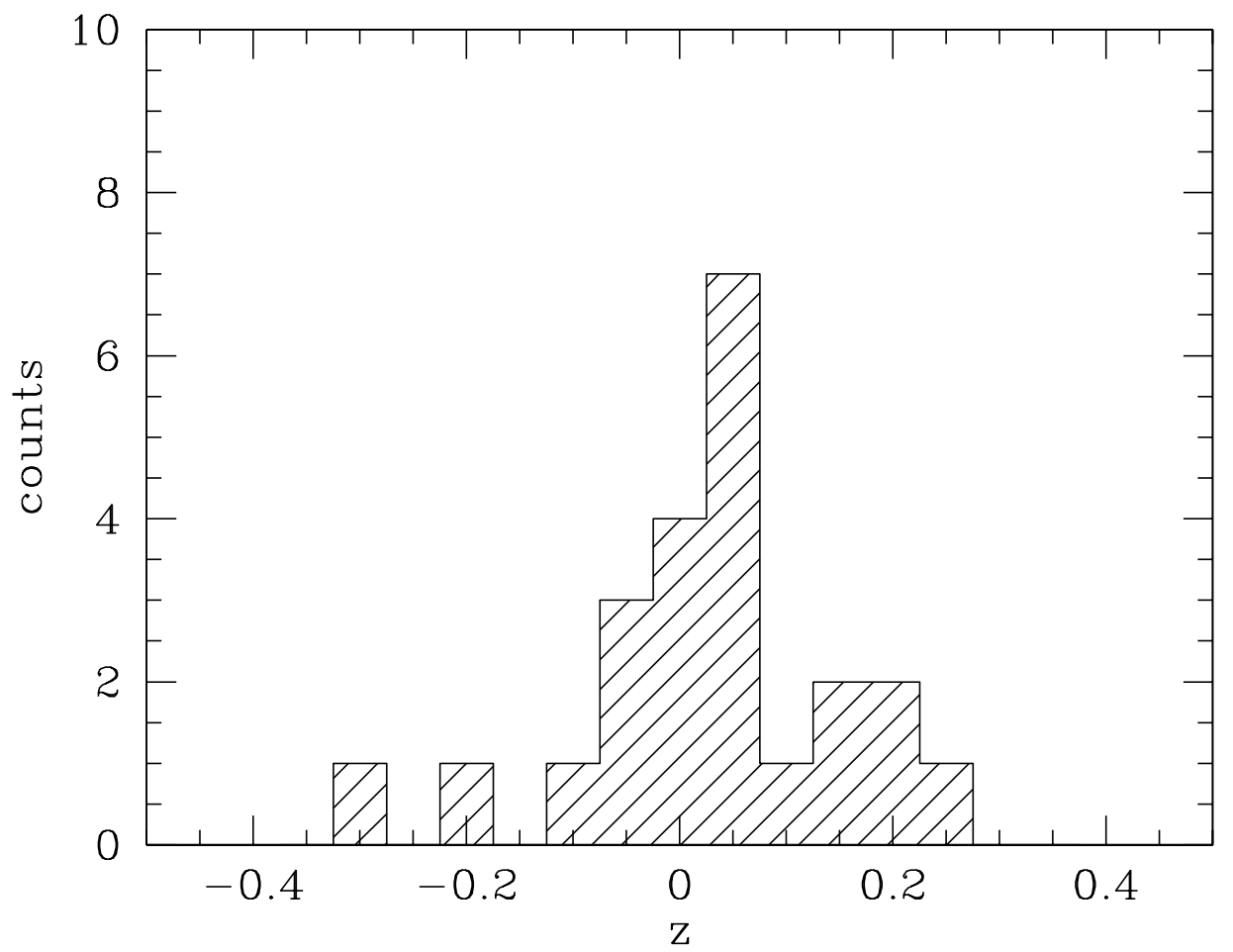

Fig. 7.- Histogram of "redshifts" for the 23 luminous infrared starbursts derived using our SED template. 
Table 1. Spectral Energy Distribution Model Fits

\begin{tabular}{lcccccc}
\hline \hline \multicolumn{1}{c}{ Name } & $\begin{array}{c}\mathrm{L}(\mathrm{FIR}) \\
\left(10^{10} L_{\odot}\right)\end{array}$ & $\begin{array}{c}S F R^{\mathrm{a}} \\
\left(M_{\odot} y r^{-1}\right)\end{array}$ & $\begin{array}{c}T_{d} \\
(\mathrm{~K})\end{array}$ & $\beta^{\mathrm{b}}$ & $f_{n t h}{ }^{\mathrm{c}}$ & “ $z_{p h} "$ \\
\hline Zw049.057 & 10 & 18 & 54 & 1.30 & 0.6 & +0.06 \\
NGC 5256 & 11 & 19 & 51 & 1.35 & 4.0 & +0.07 \\
MCG+00-29-023 & 11 & 19 & 49 & 1.15 & 1.3 & +0.25 \\
NGC 3110 & 12 & 20 & 46 & 1.35 & 1.2 & +0.19 \\
Mrk 331 & 16 & 27 & 57 & 1.15 & 0.8 & +0.06 \\
NGC 1614 & 21 & 37 & 61 & 1.40 & 1.0 & -0.07 \\
UGC 2369 & 21 & 37 & 52 & 1.30 & 1.2 & +0.16 \\
NGC 2623 & 21 & 37 & 57 & 1.45 & 1.1 & -0.07 \\
Arp 193 & 25 & 42 & 47 & 1.70 & 1.5 & +0.01 \\
Arp 148 & 25 & 42 & 50 & 1.20 & 0.9 & +0.22 \\
IRAS 10173+0828 & 33 & 57 & 69 & 1.10 & 0.6 & +0.02 \\
NGC 6240 & 35 & 61 & 49 & 1.45 & 3.9 & -0.07 \\
Mrk 848 & 35 & 61 & 62 & 1.20 & 1.6 & +0.06 \\
IRAS 15250+3609 & 48 & 82 & 74 & 1.05 & 1.1 & +0.01 \\
UGC 5101 & 54 & 93 & 47 & 1.40 & 2.5 & +0.14 \\
IRAS 08572+3915 & 55 & 93 & 74 & 1.60 & 0.3 & -0.22 \\
IRAS 05189-2524 & 57 & 98 & 70 & 1.05 & 0.6 & +0.05 \\
IRAS 10565+24 & 60 & 104 & 55 & 1.55 & 0.4 & +0.06 \\
Mrk 273 & 73 & 127 & 61 & 1.40 & 1.7 & -0.10 \\
Arp 220 & 95 & 163 & 59 & 1.15 & 1.0 & +0.05 \\
IRAS 12112+0305 & 106 & 183 & 63 & 1.25 & 0.8 & +0.02 \\
IRAS 14348-1447 & 109 & 187 & 63 & 1.50 & 1.0 & +0.09 \\
Mrk 231 & 137 & 236 & 72 & 1.35 & 2.0 & -0.30 \\
& & & & & & \\
\hline & & & & & & \\
\hline
\end{tabular}

${ }^{\mathrm{a}} S F R=L_{F I R} /\left(5.8 \times 10^{9} L_{\odot}\right) M_{\odot} \mathrm{yr}^{-1}$ (Kennicutt 1998)

${ }^{\mathrm{b}}$ Dust emissivity.

${ }^{\mathrm{c}}$ Multiplicative correction factor for non-thermal radio continuum emission. The $f_{n t h}=1.0$ corresponds to the Galactic normalization adopted by Condon (1992). 
Table 2. Redshift Estimates for Submm Sources

\begin{tabular}{|c|c|c|c|c|c|c|c|}
\hline Name & $z_{s p}$ & $z_{S I}$ & $z_{p h}$ & $S F R^{\mathrm{a}}$ & $N_{t o t}\left(N_{d e t}\right)^{\mathrm{b}}$ & $\chi_{n}^{2 \mathrm{c}}$ & Refs. \\
\hline \multicolumn{8}{|l|}{ Known Redshifts: } \\
\hline CUDSS14.18 & 0.66 & $1.12_{-0.45}^{+0.53}$ & $1.11 \pm 0.21$ & 200 & $5(3)$ & 0.02 & 1 \\
\hline SMM J02399-0134 & 1.06 & $1.17_{-0.45}^{+0.53}$ & $0.93 \pm 0.19$ & 525 & $5(3)$ & 0.5 & 2 \\
\hline HR10 & 1.44 & $1.49_{-0.53}^{+0.75}$ & $1.75 \pm 0.28$ & 380 & $7(5)$ & 0.7 & $3,4,5$ \\
\hline SMM J14011+0252 & 2.57 & $2.53_{-0.82}^{+1.24}$ & $2.73 \pm 0.37$ & 850 & $5(4)$ & 1.0 & 2,6 \\
\hline SMM J02399-0136 & 2.80 & $1.65_{-0.53}^{+0.80}$ & $2.83 \pm 0.38$ & 1400 & $9(5)$ & 28 & 2,7 \\
\hline \multicolumn{8}{|l|}{ No Known Redshifts: } \\
\hline FIRBACK J1608+5418 & - & $0.05_{-0.05}^{+0.21}$ & $0.84 \pm 0.18$ & 690 & $7(5)$ & 17 & $12,13,14$ \\
\hline SMM J14009+0252 & - & $1.27_{-0.54}^{+0.59}$ & $1.30 \pm 0.23$ & 980 & $5(4)$ & 2.3 & 2,6 \\
\hline CUDSS14.1 & - & $2.01_{-0.71}^{+1.10}$ & $2.06 \pm 0.31$ & 520 & $7(4)$ & 0.5 & 10,11 \\
\hline Lockman850.1 & - & $2.95_{-0.98}^{+1.49}$ & $2.72 \pm 0.37$ & 570 & $8(5)$ & 0.2 & 15 \\
\hline SMM J00266+1708 & - & $3.49_{-1.23}^{+2.03}$ & $3.50 \pm 0.45$ & 1200 & $6(4)$ & 0.2 & 2 \\
\hline SMM J09429+4658 & - & $\geq 3.6$ & $3.86 \pm 0.49$ & 890 & $4(4)$ & 4.0 & 2,6 \\
\hline HDF850.1 & - & $>2.6$ & $4.11 \pm 0.51$ & 380 & $8(4)$ & 0.8 & 8,9 \\
\hline \multicolumn{8}{|l|}{ QSOs: } \\
\hline FSC $10214+4724$ & 2.28 & $1.49_{-0.51}^{+0.74}$ & $1.51 \pm 0.25$ & 2600 & $9(9)$ & 7.6 & 16,17 \\
\hline H $1413+117$ & 2.56 & $0.88_{-0.34}^{+0.41}$ & $0.31 \pm 0.13$ & 850 & $9(9)$ & 28 & 17,18 \\
\hline LBQS $1230+1627$ & 2.74 & $2.19_{-0.75}^{+1.16}$ & $2.66 \pm 0.37$ & 1300 & $4(4)$ & 0.5 & $19,20,21$ \\
\hline SMM J04135+1027 & 2.83 & $\geq 0.9$ & $1.83 \pm 0.28$ & 1560 & $4(3)$ & 0.8 & 22 \\
\hline APM $08279+5255$ & 3.87 & $2.18_{-0.71}^{+1.10}$ & $1.74 \pm 0.28$ & 4900 & $7(7)$ & 9.4 & $23,24,25$ \\
\hline BR $1335-0417$ & 4.40 & $1.81_{-0.62}^{+0.95}$ & $2.84 \pm 0.38$ & 1200 & $5(5)$ & 7.0 & $17,19,20,21,26$ \\
\hline BR $0952-0115$ & 4.43 & $1.35_{-0.46}^{+0.63}$ & $0.96 \pm 0.20$ & 460 & $5(4)$ & 5.6 & $19,20,21,26$ \\
\hline BR $1202-0725$ & 4.70 & $3.13_{-1.14}^{+1.81}$ & $3.20 \pm 0.42$ & 2300 & $11(9)$ & 3.1 & $17,19,20,21,26$ \\
\hline
\end{tabular}

${ }^{\mathrm{a}} S F R$ is in $M_{\odot} \mathrm{yr}^{-1}$.

${ }^{\mathrm{b}} N_{\text {tot }}$ and $N_{\text {det }}$ are total number of SED data used for the fit and the number of detections (i.e. $N_{\text {tot }}=$ $\left.N_{\text {det }}+N_{\text {limits }}\right)$.

${ }^{\mathrm{c}} \chi_{n}^{2} \equiv \chi^{2} / N_{d e t}$.

References. - (1) Eales et al. (2001); (2) Smail et al. (2000); (3) Cimatti et al. (1998); (4) Dey et al. (1999); (5) Andreani et al. (2000); (6) Ivison et al. (2000); (7) Ivison et al. (1998); (8) Hughes et al. (1998); (9) Downes et al. (1999a); (10) Eales et al. (2000); (11) Gear et al. (2000); (12) Benford (1999); (13) Scott et al. (2000); (14) Dole et al. (2001); (15) Lutz et al. (2001); (16) Rowan-Robinson et al. (1993); (17) Benford et al. (1999); (18) Barvainis et al. (1992); (19) Omont et al. (1996); (20) Guilloteau et al. (1999); (21) Yun et al. (2000); (22) Knudsen et al. (2000); (23) Irwin et al. (1998); (24) Lewis et al. (1998); (25) Downes et al. (1999b); (26) McMahon et al. (1999) 